\title{
Facile Synthesis of Mesoporous Graphene Materials from Potassium Humate for Supercapacitor Electrodes
}

\author{
Guangxu Huang, ${ }^{1,2,3}$ Qianhao Geng $\mathbb{D},{ }^{1}$ Weiwei Kang, ${ }^{4}$ Yingbin Liu $\mathbb{D},{ }^{1}$ Bin Duan, \\ and Chuanxiang Zhang ${ }_{(D)}^{1,2,3}$
}

${ }^{1}$ College of Chemistry and Chemical Engineering, Henan Polytechnic University, Jiaozuo 454000, China

${ }^{2}$ Collaborative Innovation Center of Coal Work Safety of Henan Province, Jiaozuo 454003, China

${ }^{3}$ Henan Key Laboratory of Coal Green Conversion, Jiaozuo 454003, China

${ }^{4}$ College of Chemistry and Chemical Engineering, Southeast University, Nanjing 211189, China

${ }^{5}$ Do-Fluoride Chemicals Co., Ltd., Jiaozuo 454150, China

Correspondence should be addressed to Chuanxiang Zhang; zcx223@163.com

Received 5 March 2018; Accepted 24 June 2018; Published 24 July 2018

Academic Editor: Miguel A. Domínguez-Crespo

Copyright (c) 2018 Guangxu Huang et al. This is an open access article distributed under the Creative Commons Attribution License, which permits unrestricted use, distribution, and reproduction in any medium, provided the original work is properly cited.

\begin{abstract}
Mesoporous graphene materials (MG-X) were prepared from potassium humate coupled with magnesium acetate. The freezedrying pretreatment and $\mathrm{MgO}$ particles not only prevent the agglomeration of graphene sheets and form mesopores but also help to reduce the oxygen content and enlarge the $\mathrm{sp}^{2}$ carbon domain of MG-X. MG-X possess optimized characteristics including large specific surface area $\left(>1100 \mathrm{~m}^{2} \cdot \mathrm{g}^{-1}\right)$, high mesoporosity $(\sim 90 \%)$, and low oxygen content $(\sim 7 \%)$. When used as supercapacitor electrodes, MG-X demonstrate optimal electrochemical performances among all materials prepared here, such as high gravimetric capacitance $\left(150 \mathrm{~F} \cdot \mathrm{g}^{-1}\right.$ at high current density of $3.3 \mathrm{~A} \cdot \mathrm{g}^{-1}$ ) and enhanced rate capability with the capacitance retention of $\sim 80 \%$ from 0.041 to $3.3 \mathrm{~A} \cdot \mathrm{g}^{-1}$. Moreover, MG-5 exhibits good cycling stability with a capacitance retention of $98.6 \%$ after 1000 cycles at $2.46 \mathrm{~A} \cdot \mathrm{g}^{-1}$. This work provides a facile and efficient preparation method for mesoporous graphene materials used as supercapacitor electrodes.
\end{abstract}

\section{Introduction}

As an advanced energy storage device, supercapacitors have attracted considerable attention in recent years due to their excellent properties such as high rate capability and long cycle life $[1,2]$. Up to now, the electrodes used in most commercial supercapacitors are made of carbon materials [3]. Based on the energy storage mechanism of supercapacitors, it is of great significance to design and produce carbon materials with a combination of high specific surface area, suitable pore size distribution, and good electrical conductivity. Graphene, a single atomic layer of graphite consisting of $\mathrm{sp}^{2}$-hybridized carbon atoms in a honeycomb lattice [4], is particularly suitable for supercapacitor applications because of its excellent characteristics including high specific surface area and superb electronical conductivity [5]. However, graphene sheets easily suffer from unavoidable restacking owing to the strong $\pi-\pi$ interactions between graphene layers during preparation and subsequent processing $[1,6]$. Consequently, some unique and excellent properties that individual graphene sheets possess such as high specific surface area and peculiar electron transport behaviors are significantly compromised or even unavailable in an assembly [7]. Therefore, the actual specific capacitances of graphene-based supercapacitor electrodes are usually lower than the theoretical values $[8,9]$. It was found that incorporating pores into the graphene architectures to fabricate porous graphene materials can provide a good solution to the issue of aggregation or sheet-to-sheet restacking $[10,11]$. In comparison with micropores and macropores, mesopores in graphene are more 
beneficial to enhance electrochemical performance of the corresponding electrodes by serving as an expressway for electrolyte ion diffusion and providing low resistances for charge transfer $[6,12]$.

Currently, the most promising approach to synthesize graphene at a large scale involves the ultrasonic exfoliation of graphite oxide to generate graphene oxide and followed by reduction to restore the $\mathrm{sp}^{2}$ carbon network. Moreover, graphite oxide is typically synthesized from graphite powders by harsh oxidation based on potassium permanganate and concentrated sulfuric acid according to the Hummers method. For the preparation of mesoporous graphene materials, some efforts have been demonstrated by various strategies, in which the graphite oxide or graphene oxide were usually used as the starting materials $[2,13,14]$. According to the facts described above, there are three factors that should be considered for the synthesis of mesoporous graphene materials-the preparation of graphite oxide or graphene oxide, the formation of mesopores, and the reduction process. Evidently, such synthesis strategy of mesoporous graphene materials is potentially plagued by a tedious synthesis process, environmental concern, and even explosion risk [15]. It is very imperative to develop facile and efficient approaches to synthesize mesoporous graphene materials for supercapacitor applications.

Humic acid, a natural graphite oxide-like material [16] containing a number of oxygenated groups around the edges of the graphene-like core, has been used as a precursor to prepare graphene-related materials $[17,18]$. Water-soluble potassium humate is a neutralized product of insoluble humic acid and $\mathrm{KOH}$. Potassium humate has been adopted as a graphite oxide-like material to prepare reduced graphite oxide for supercapacitor electrode material [19]. Herein, we present a facile synthesis of mesoporous graphene materials via freeze drying followed by carbonizing the mixture of potassium humate and magnesium acetate. The solubility advantage of potassium humate and magnesium acetate in water can be taken to realize their homogenous mixing in nanoscale, which further helps to incorporate $\mathrm{MgO}$ particles among graphene layers. Furthermore, those three issues influencing the synthesis of mesoporous graphene materials can be addressed facilely and simultaneously. The morphology, microstructure, and composition of the as-prepared mesoporous graphene materials were investigated in detail. The electrochemical performances of the corresponding supercapacitor electrodes were also evaluated in terms of galvanostatic charge-discharge, cyclic voltammetry, electrochemical impedance, and cycling performance.

\section{Experimental}

2.1. Material Preparation. All chemical reagents used in this experiment are of analytical grade without any further purification. The potassium humate and magnesium acetate were purchased from Yiliang humic acid Co. and Shanghai Chemical Co. in China, respectively. Magnesium acetate and potassium humate (with the mass ratios of 1, 3, and 5) were dissolved in $400 \mathrm{ml}$ deionized water, followed by stirring overnight and subsequent freeze drying. The resultant solid mixtures were directly carbonized in a tube furnace at $800^{\circ} \mathrm{C}$ for an hour with a heating rate of $5^{\circ} \mathrm{C} \cdot \mathrm{min}^{-1}$ under nitrogen atmosphere. After cooling naturally to room temperature, the carbonized materials were washed with diluted hydrochloric acid, rinsed with deionized water until $\mathrm{pH}=7$, and dried at $110^{\circ} \mathrm{C}$ for $2 \mathrm{~h}$. The obtained mesoporous graphene materials were nominated as MG-X, where $\mathrm{X}$ stands for the mass ratio of magnesium acetate to potassium humate correspondingly. For comparison, $\mathrm{MC}$ was prepared following the same procedure without the addition of magnesium acetate and MC-X were obtained without the freeze-drying process.

2.2. Characterization. The morphology of as-prepared materials was observed by scanning electron microscope (SEM, JSM-6390LV, and JEOL) and transmission electron microscopy (TEM, JEM-2100, and JEOL). $\mathrm{N}_{2}$ adsorptiondesorption isotherms were measured using a Quantachrome Autosorb-iQ-MP analyzer at $77 \mathrm{~K}$. The specific surface area $\left(S_{\mathrm{BET}}\right)$ was calculated from the isotherm using the Brunauer-Emmett-Teller method, and the total pore volume $\left(V_{\mathrm{t}}\right)$ was determined according to the $\mathrm{N}_{2}$ adsorption at the relative pressure $\left(P / P_{0}\right)$ of 0.99 . The micropore volume $\left(V_{\text {mic }}\right)$ was determined by the t-plot method, and the mesopore volume $\left(V_{\text {mes }}\right)$ was calculated by the difference of total pore volume and micropore volume. Before the test, the samples were degassed at $200^{\circ} \mathrm{C}$ for 10 hours to remove the water vapor and other foreign gas matters. X-ray photoelectron spectroscopy (XPS) (Thermo ESCALAB 250Xi) was used to investigate the surface chemical composition. Raman spectra were obtained with an inVia Ar ion laser (Renishaw, UK) using a $520 \mathrm{~nm}$ exciting radiation.

2.3. Electrochemical Measurements. The electrodes were prepared by pressing a homogeneous mixture of mesoporous graphene materials (85 wt.\%), black carbon (10 wt.\%), and polytetrafluoroethylene ( $5 \mathrm{wt} . \%)$ under the pressure of $10 \mathrm{MPa}$. A sandwich two-electrode system was used to test the galvanostatic charge/discharge (GCD), cyclic voltammetry $(\mathrm{CV})$, and cycle performance in $3 \mathrm{~mol} \cdot \mathrm{L}^{-1} \mathrm{KOH}$ electrolyte by the supercapacitor testing system (SCTS, Arbin, USA). The GCD was performed with the voltage varying from 0.05 to $0.9 \mathrm{~V}$ and the current from 0.85 to $85 \mathrm{~mA}$. The gravimetric capacitances $\left(\mathrm{F} \cdot \mathrm{g}^{-1}\right)$ were calculated from the following:

$$
C_{m}=2(I \times \Delta t) \div(m \times \Delta V),
$$

where $I(\mathrm{~A})$ is the constant current, $m(\mathrm{~g})$ is the mass of the active material in the single electrode, and $\triangle t / \triangle V$ is calculated from the slope of the discharge curve. The CV measurements were conducted at different scan rates between 1 and $20 \mathrm{mV} \cdot \mathrm{s}^{-1}$. The electrochemical impedance spectroscopy (EIS) was recorded at frequencies from $100 \mathrm{kHz}$ to $1 \mathrm{mHz}$ with the electrochemical test system (Parstat2273, USA).

\section{Results and Discussion}

As shown in Figure 1(a), MG-1 exhibits bulk amorphous structure with some thin layers at the edge (see red rectangle in Figure 1(a)). Figure 1(b) shows that MG-3 displays three- 


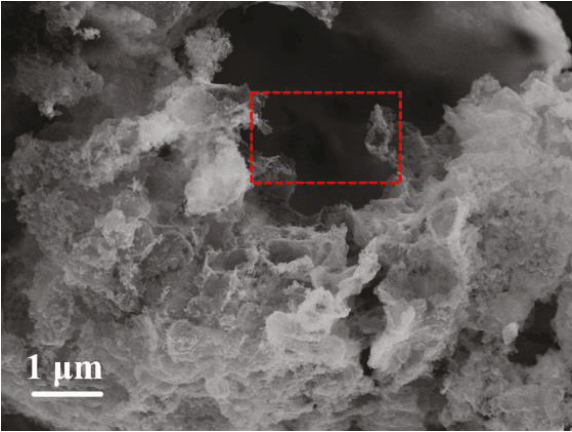

(a)

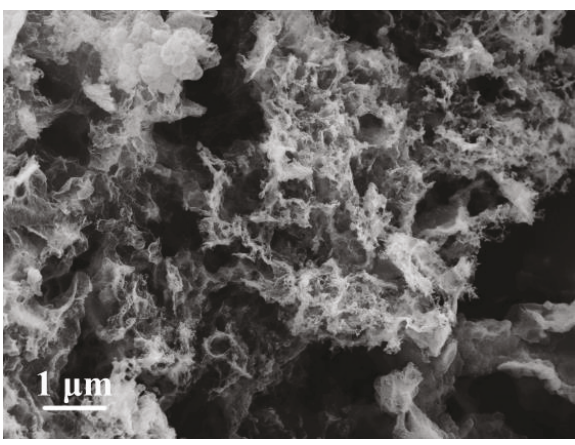

(c)

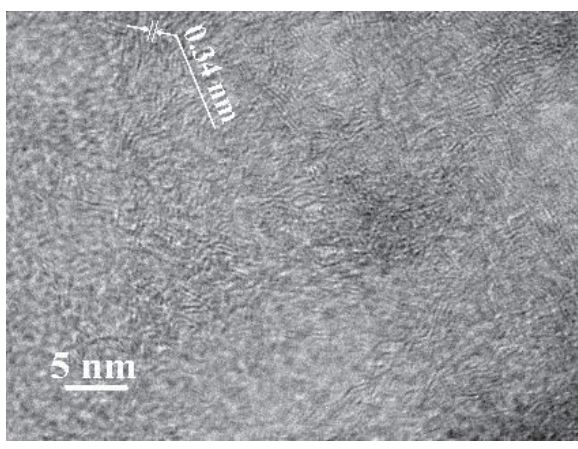

(e)

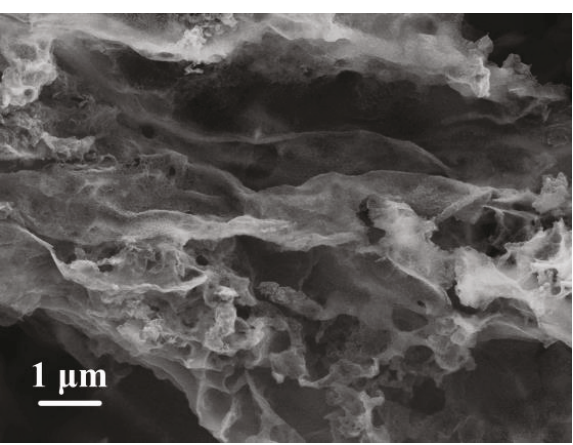

(b)

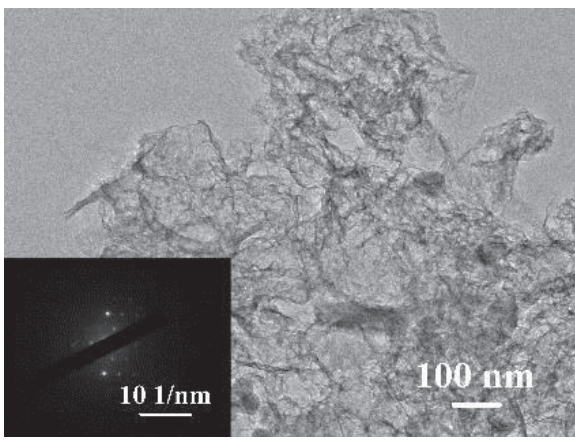

(d)

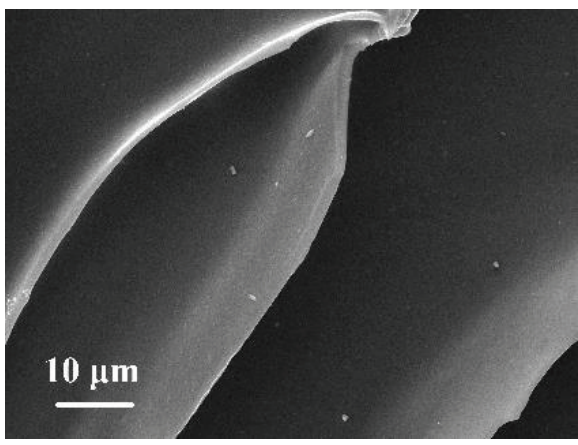

(f)

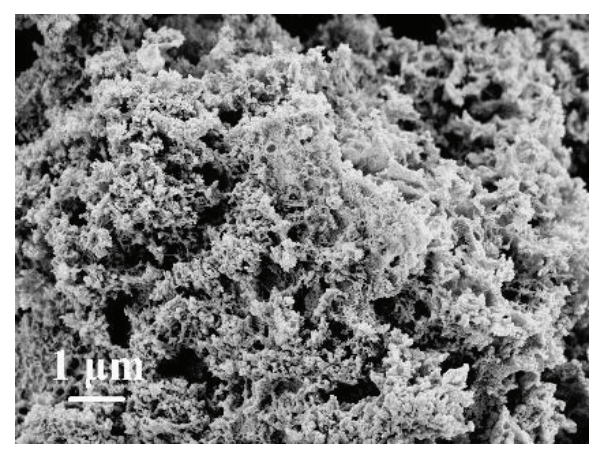

(g)

Figure 1: SEM images of MG-1 (a), MG-3 (b), and MG-5 (c). TEM image with SAED pattern (d); high-resolution TEM image (e) of MG-5; and SEM image of MC (f) and MC-3 (g).

dimensional (3D) loose and lamellar structure with the silky and wavy surface, suggesting the formation of porous graphene sheets. MG-5 shows an interconnected, wrinkled, and porous framework, which is mainly fabricated by thin graphene sheets (Figure $1(\mathrm{c})$ ) and similar to the $3 \mathrm{D}$ mesoporous graphene [20]. The TEM image in Figure 1(d) indicates that MG-5 has a rough surface with intrinsic wrinkles and lots of interconnected nanopores on the surface, similar to the $3 \mathrm{D}$ porous graphene reported [21]. The selected area electron diffraction pattern (SAED) exhibits a 


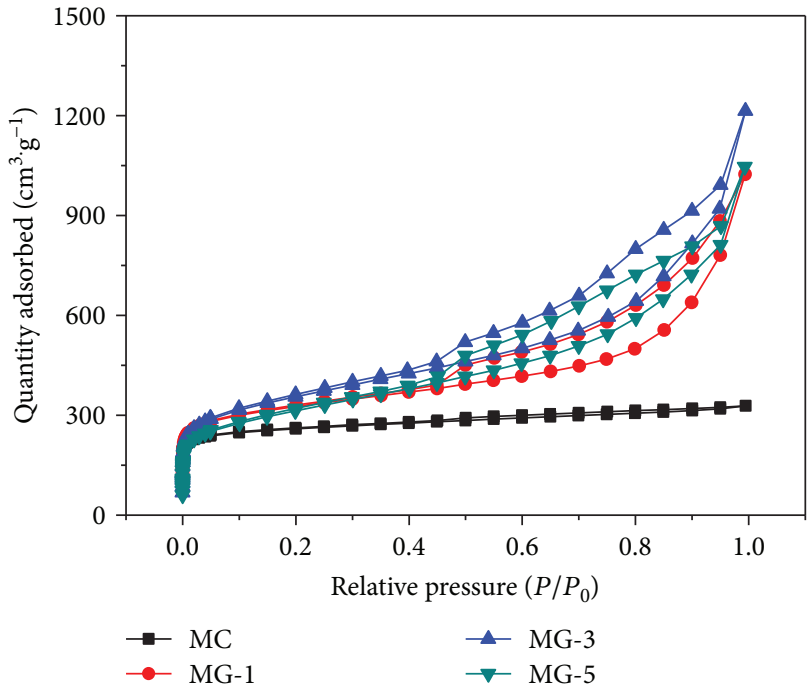

(a)

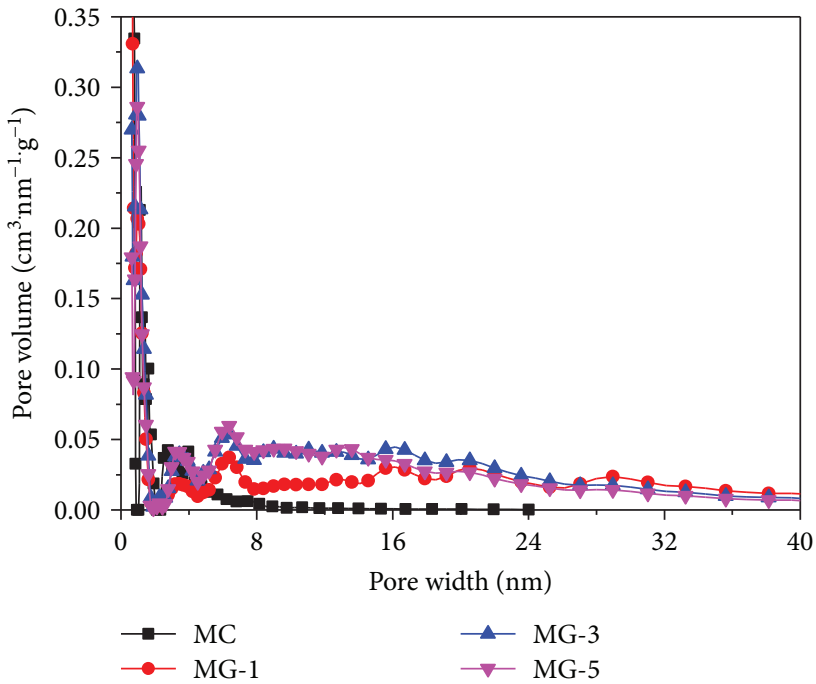

(b)

Figure 2: $\mathrm{N}_{2}$ adsorption-desorption isotherms (a) and pore size distribution curves (b) of MG-X.

TABle 1: Pore structure parameters of MG-X.

\begin{tabular}{lcccccc}
\hline Samples & $S_{\mathrm{BET}}\left(\mathrm{m}^{2} \cdot \mathrm{g}^{-1}\right)$ & $V_{\mathrm{t}}\left(\mathrm{cm}^{3} \cdot \mathrm{g}^{-1}\right)$ & $V_{\text {mic }}\left(\mathrm{cm}^{3} \cdot \mathrm{g}^{-1}\right)$ & $V_{\text {mes }}\left(\mathrm{cm}^{3} \cdot \mathrm{g}^{-1}\right)$ & $V_{\text {mes }} / V_{\mathrm{t}}(\%)$ & 36.8 \\
\hline MC & 997 & 0.51 & 0.32 & 0.19 & 1.30 & 81.6 \\
MG-1 & 1188 & 1.59 & 0.29 & 1.67 & 8.78 \\
MG-3 & 1267 & 1.88 & 0.21 & 1.47 & 9.4 \\
MG-5 & 1112 & 1.62 & 0.16 & 90.4 & 9.40 \\
\hline
\end{tabular}

perfect hexagonal pattern (inset of Figure 1(d)), demonstrating the graphitic crystalline nature and few-layer graphene feature of MG-5 [22, 23]. Figure 1(e) shows a highresolution TEM image of MG-5, in which few-layer graphene sheets with clear lattice structure were observed. The interplanar distance was measured to be $\sim 0.34 \mathrm{~nm}$ corresponding to $\left(\begin{array}{lll}0 & 0 & 2\end{array}\right)$ planes of graphitic carbon, confirming its good crystallinity [24]. However, MC presents micron-level-thick sheets with smooth surface (Figure 1(f)), suggesting the stacking configuration of graphene sheets without the addition of magnesium acetate. Moreover, MC-3 demonstrates a porous structure having no apparent thin graphene sheets (Figure $1(\mathrm{~g})$ ), only indicating the pore-forming effect of the $\mathrm{MgO}$ template. Therefore, the $\mathrm{MgO}$ particles derived from magnesium acetate and freeze-drying pretreatment both play key roles in preventing the restacking of graphene layers and generating pores in $\mathrm{MG}-\mathrm{X}$.

The nitrogen adsorption-desorption isotherms of MG-X exhibit combined characteristics of type I and type IV (Figure 2(a)) [25], the apparent hysteresis loops suggest the dominant presence of mesopores. MC displays a steep uptake below $P / P_{0}=0.01$ and subsequent saturated adsorption platform, indicating the existence of micropores in large quantity $[26,27]$. Figure 2(b) shows the pore size distribution (PSD) curves, MG-X display almost the same PSD with the MC's, the pore width range is less than $5 \mathrm{~nm}$, while a remarkable increase happened to the pore volume that the relative pore width range is larger than $5 \mathrm{~nm}$. The pore structure parameters are summarized in Table 1, MG-X have higher $S_{\mathrm{BET}}$ $\left(1100 \sim 1300 \mathrm{~m}^{2} \cdot \mathrm{g}^{-1}\right)$ and much larger total pore volume, mesopore volume, and mesoporosity (reaching up to $81.6 \%$, $88.7 \%$, and $90.4 \%$, resp.) than MC's. Specifically, the $S_{\mathrm{BET}}$ and mesoporosity of MG-X are also higher than those of the mesoporous graphene materials reported [15, 28, 29]. Moreover, MC-X demonstrate narrower mesopore size distribution and lower $S_{\mathrm{BET}}$ (see Figure S1a, Figure S1b, and Table S1 in the Supplementary Materials) than those of MG-X. These results confirm that the MgO particles and freeze-drying pretreatment both contribute to the formation of pores and result in higher $S_{\mathrm{BET}}$. The SEM, TEM, and nitrogen adsorption-desorption results indicate that the mesoporous graphene materials can be synthesized from potassium humate coupled with magnesium acetate via freeze-drying followed by simple carbonization. MG-X possess higher mesoporosity than $\mathrm{MC}$ and larger $S_{\mathrm{BET}}$ than $\mathrm{MC}-\mathrm{X}$, which make them more promising to electrodes because of considerable specific capacitance and better rate capability [5].

It can be seen from the XPS spectra that MG-X and MC all possess two main peaks at about 285 and $533 \mathrm{eV}$ (Figure 3(a)), which were assigned to the $\mathrm{C} 1 \mathrm{~s}$ and $\mathrm{O} 1 \mathrm{~s}$ components, respectively. The $\mathrm{O} / \mathrm{C}$ atomic ratio of $\mathrm{MC}(0.26)$ is much higher than those of MG-1 (0.10), MG-3, and MG-5 (both of them are 0.08 ), suggesting that the $\mathrm{MgO}$ particles 


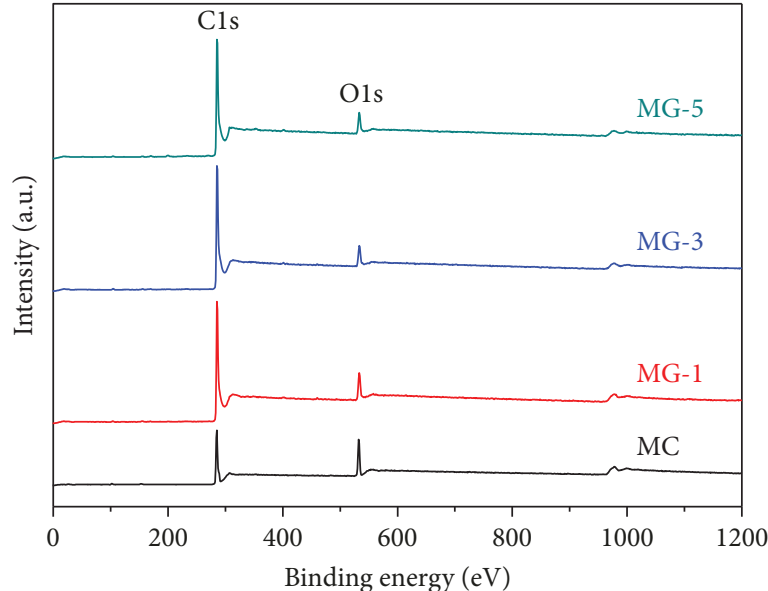

(a)

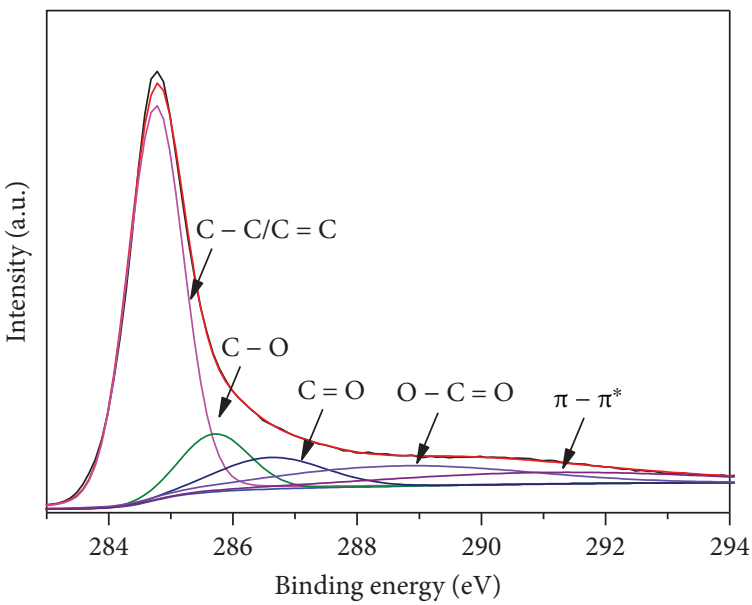

(c)

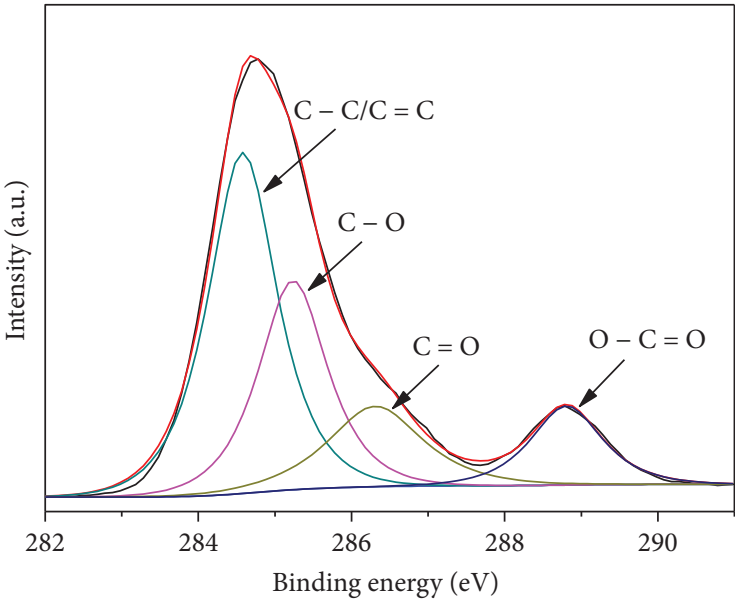

(b)

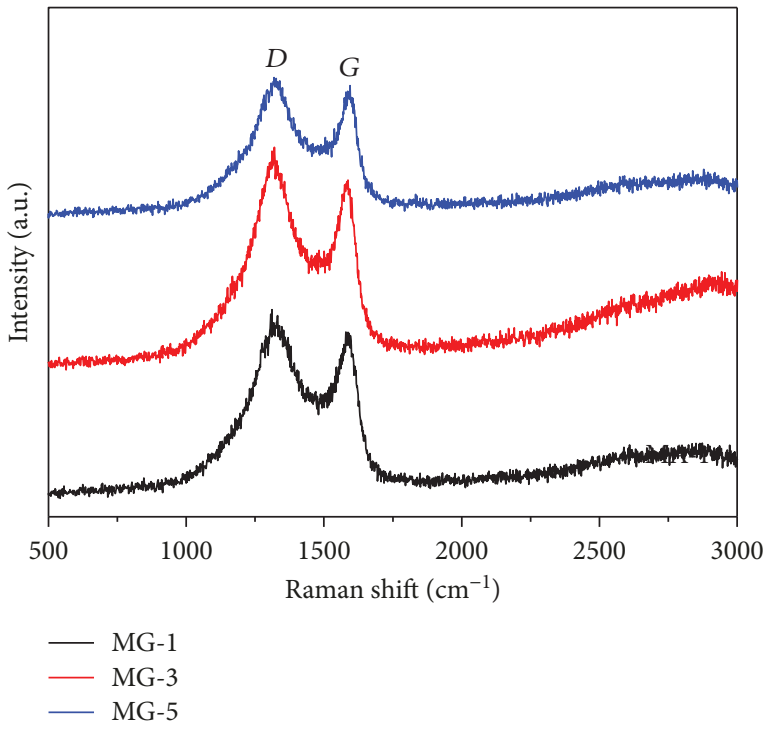

(d)

FIGURE 3: Full-range XPS spectra of MG-X and MC (a), C1s XPS spectra of MC (b), C1s XPS spectra of MG-3 (c), and Raman spectra of MG-X (d).

induced catalytic reduction of carbon materials. According to the curve fitting of C1s spectra (Figures 3(b) and 3(c)), compared with MC, MG-3 presents enhanced nonoxygenated carbon peaks $(C=C / C-C)$ and weakened oxygenated carbon peaks $(\mathrm{C}-\mathrm{OH} / \mathrm{C}-\mathrm{O}-\mathrm{C}, \mathrm{C}=\mathrm{O}$, and $\mathrm{O}-\mathrm{C}=\mathrm{O})$, confirming such reduction process. More importantly, MG-3 exhibits broadened tail with an additional peak component at $291.4 \mathrm{eV}$ on account of $\pi-\pi^{*} \mathrm{sp}^{2}$ transition, revealing the creation of more aromatic rings in the carbon basal plane $[30,31]$. In fact, $\mathrm{MgO}$ nanoparticles can increase the graphitic nature of the layered reduced graphene oxide microstructure [32]. The relatively low oxygen content and enhanced $\mathrm{sp}^{2}$ carbon domain can improve the electrical conductivity of MG-X, which is closely related to their electrochemical performances. However, the MC-1, MC-3, and MC-5 have O/C atomic ratios of $0.39,0.14$, and 0.10 , respectively, which are higher than those of MG-X. Thus, the freeze-drying pretreatment can also help to reduce the oxygen content of MG-X, which is mainly due to the looser and thinner graphene nanosheets [33]. To the best of our knowledge, there are few reports about the influences of freeze-drying pretreatment on the reduction of graphite oxide or graphene oxide. More importantly, the reduction reactions took place without any additional reductant and catalyst. The Raman spectra of MG-X exhibit D bands at around $1346 \mathrm{~cm}^{-1}$ and $\mathrm{G}$ bands at around $1580 \mathrm{~cm}^{-1}$ (Figure 3(d)), which correspond to disorder carbons (or defects) and graphitic carbons, respectively [24]. According to Figure S2 in the Supplementary Materials, the $I_{\mathrm{D}} / I_{\mathrm{G}}$ ratios of MG-1, MG-3, and MG-5 are 2.3, 2.7, and 2.6, respectively, higher than that of MC (1.1). And this result may arise from the smaller size of the newly formed graphitic domains in MG-X [5]. All in all, those three issues influencing the synthesis of mesoporous graphene materials-the graphite oxide preparation, mesopores formation, and reduction process-were addressed facilely and simultaneously. 


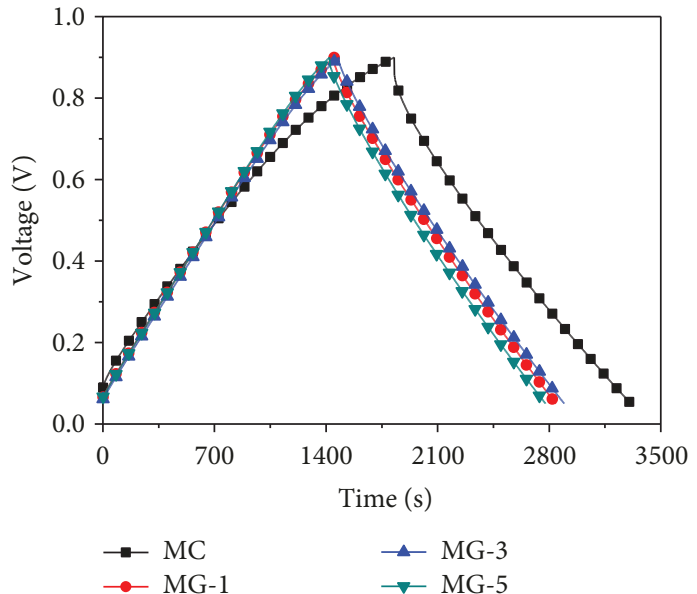

(a)

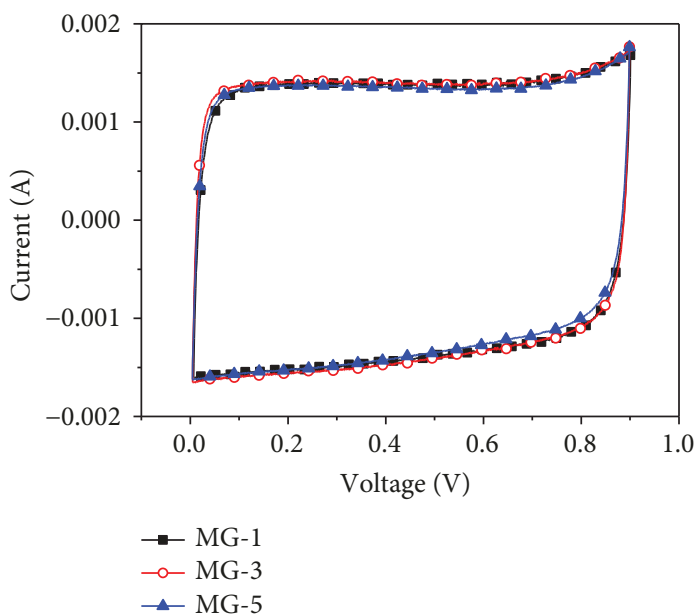

(c)

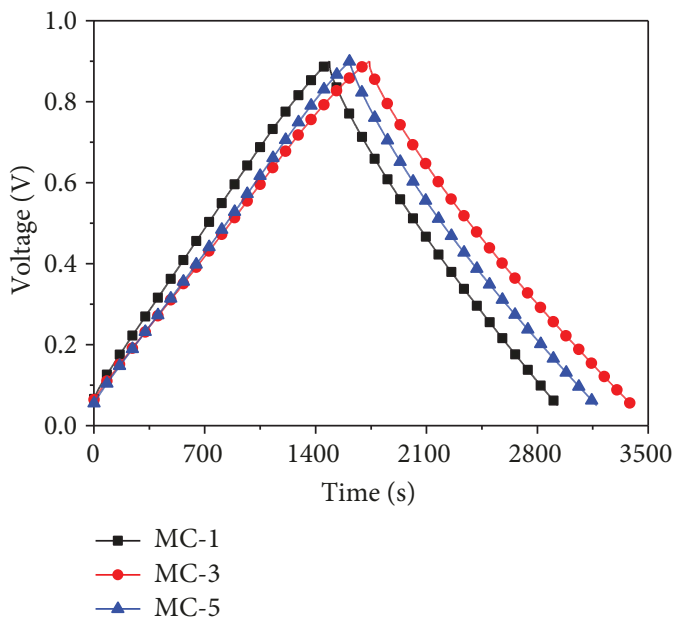

(e)

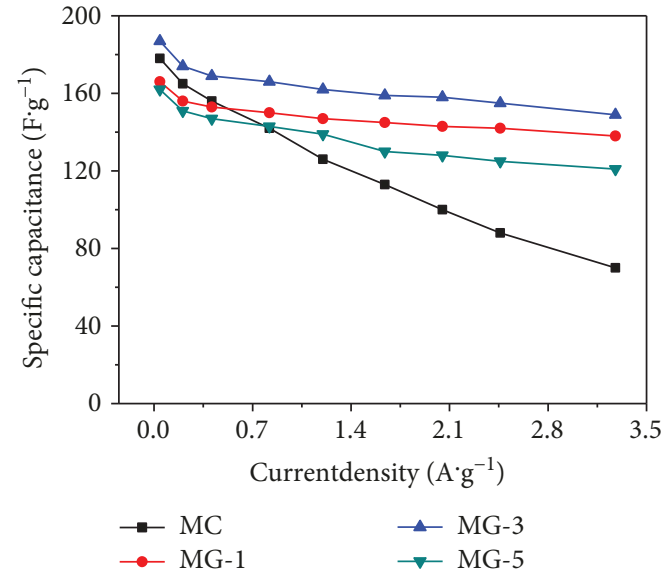

(b)

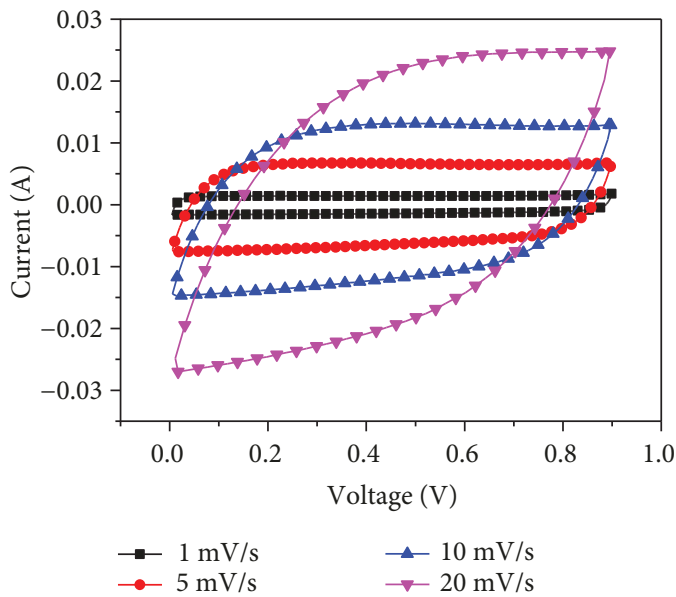

(d)

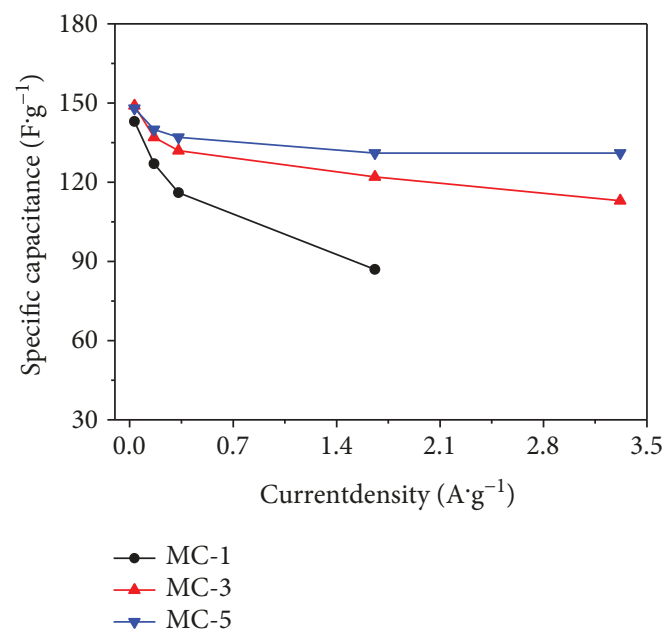

(f)

FIGURE 4: GCD curves of MG-X and MC at the current density of $0.041 \mathrm{~A} \cdot \mathrm{g}^{-1}$ (a). Rate capabilities of MG-X and MC (b), CV profiles of MG-X at the scan rate of $1 \mathrm{mV} \cdot \mathrm{s}^{-1}(\mathrm{c}), \mathrm{CV}$ profiles of MG-3 at different scan rates (d), GCD curves at the current density of $0.033 \mathrm{~A} \cdot \mathrm{g}^{-1}(\mathrm{e})$, and rate capabilities (f) of MC-X.

As shown in Figure 4(a), the GCD curves of MG-X at the current density of $0.041 \mathrm{~A} \cdot \mathrm{g}^{-1}$ are highly linear and symmetrical, indicating that the corresponding electrode materials have excellent capacitive behavior and electrochemical reversibility [34]. However, MC exhibits an obvious voltage drop at the beginning of discharge, suggesting inferior 


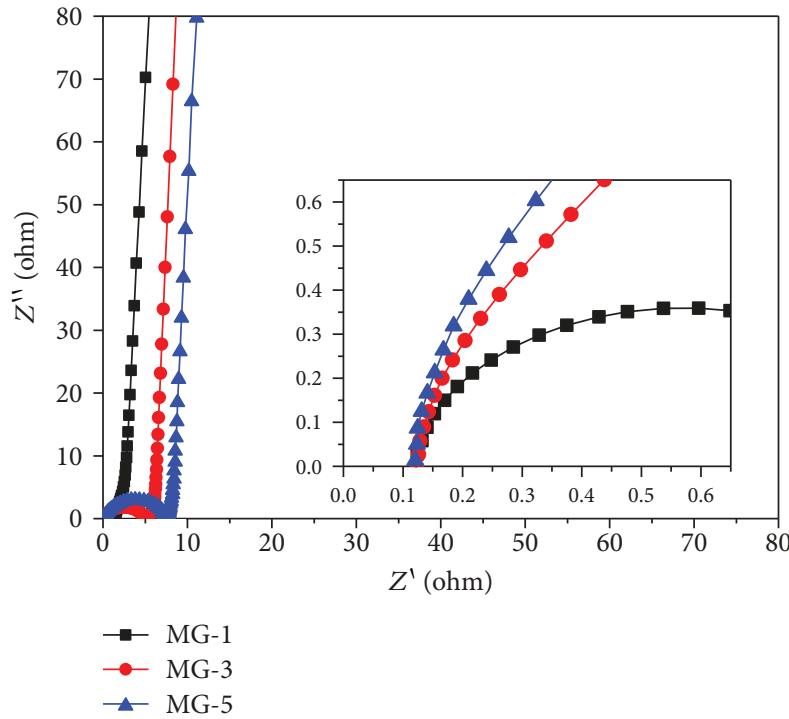

(a)

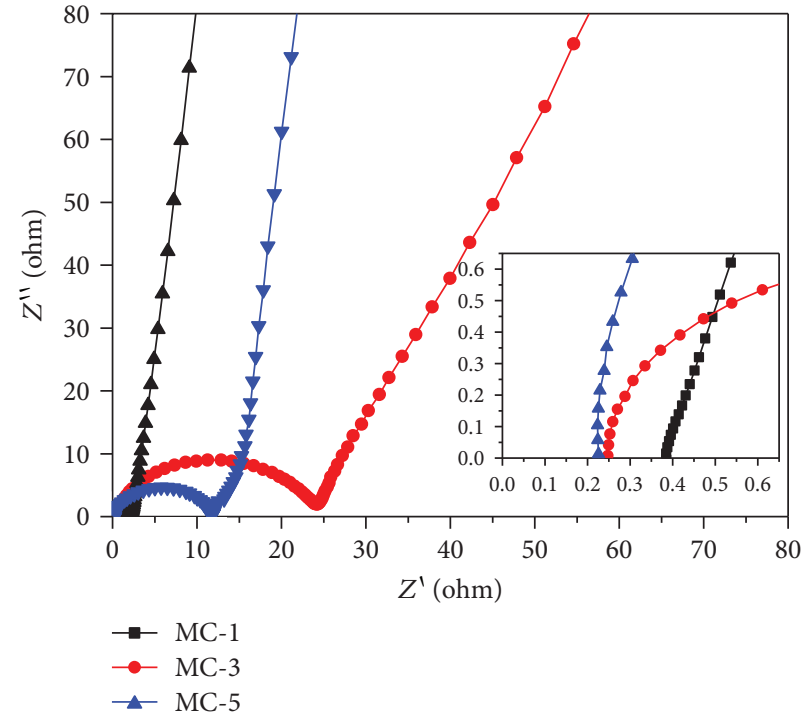

(b)

Figure 5: The Nyquist plots of MG-X as electrodes (a) and MC-X as electrodes (b).

electrochemical performance with relatively high internal resistance, which is due to its high oxygen content [35]. Based on the GCD curves, the specific capacitances of MG-1, MG-3, and MG-5 calculated from (1) at $0.041 \mathrm{~A} \cdot \mathrm{g}^{-1}$ are 166,187 , and $162 \mathrm{~F} \cdot \mathrm{g}^{-1}$, respectively, and the variation tendency is consistent with that of $S_{\mathrm{BET}}$. The considerable specific capacitances of MG-X are higher than those of some other reported mesoporous graphene materials, such as mesoporous freestanding-reduced graphene oxide-based films [14] and mesoporous-reduced graphene oxide [2]. The dependence of specific capacitance on current density is shown in Figure 4(b); MG-X exhibit excellent rate capabilities with the capacitance retention of $83 \%$ (MG-1), $80 \%$ (MG-3), and $75 \%$ (MG-5) at a higher current density of $3.3 \mathrm{~A} \cdot \mathrm{g}^{-1}$. More specifically, MG-X can still deliver a high specific capacitance of $150 \mathrm{~F} \cdot \mathrm{g}^{-1}$ at $3.3 \mathrm{~A} \cdot \mathrm{g}^{-1}$. MC has a comparable capacitance to MG-X at the current density of $0.041 \mathrm{~A} \cdot \mathrm{g}^{-1}$ but undergoes a sharp decrease in capacitance with a low retention of only $39 \%$. Obviously, the improved rate capability of MG-X is attributed to the excellent mesoporous structure and high electrical conductivity resulting from low oxygen content and enhanced $\mathrm{sp}^{2}$ carbon domain. The $\mathrm{CV}$ curves of MG-X at the scan rate of $1 \mathrm{mV} \cdot \mathrm{s}^{-1}$ (Figure 4(c)) exhibit a quasirectangular shape without visible distortion, revealing a near-ideal double-layer capacitive behavior. Furthermore, the relatively ideal rectangle shape of CV curves for MG-3 can be well maintained up to $20 \mathrm{mV} \cdot \mathrm{s}^{-1}$ (Figure $4(\mathrm{~d})$ ), confirming the improved rate capability [36].

According to the GCD curves at $0.033 \mathrm{~A} \cdot \mathrm{g}^{-1}$ (Figure 4(e)), MC-X exhibit a lower specific capacitance of $140 \sim 150 \mathrm{~F} \cdot \mathrm{g}^{-1}$ because of their relatively low $S_{\mathrm{BET}}$ and electrical conductivity based on higher oxygen content. Furthermore, the three materials have an order of MC- $1<\mathrm{MC}$ $3<$ MC-5 in terms of the rate capability (Figure 4(f)), which are in accord with their oxygen content and mesoporosity. On the basis of their relatively low oxygen content and higher mesoporosity, MC-3 and MC-5 also exhibit good rate capability of $75.8 \%$ and $88.5 \%$, respectively, at a higher current density of $3.3 \mathrm{~A} \cdot \mathrm{g}^{-1}$. In summary, among all carbon materials prepared in this work, MG-X possess optimal electrochemical performances such as higher specific capacitances and better rate capability.

Electrochemical impedance spectroscopy (EIS) helps to further analyze the electrochemical behavior of the supercapacitors. Figure 5(a) illustrates the Nyquist plots for the supercapacitor with MG-X as electrodes. At the highfrequency region, the intercept at the real axis represents the solution resistance (Rs), which is derived from the intrinsic resistance of the MG-X electrodes, the resistance of the electrolyte, and the contact resistance in electrode/ current collector interface. The MG-1, MG-3, and MG-5 display the relatively low Rs value of $\sim 0.12 \Omega$, indicating good electrical conductivity $[37,38]$, which is related to their low oxygen content and enhanced $\mathrm{sp}^{2}$ carbon domain. There are semicircles in the high-frequency range in the EIS spectrogram of three electrodes, which are caused by the charge transfer resistance $\left(R_{\mathrm{ct}}\right)$. The $R_{\mathrm{ct}}$ values of the MG-1, MG-3, and MG-5 electrodes are 1, 5 , and $7.5 \Omega$, which are consistent with their rate capabilities. Obviously, the $R_{\mathrm{ct}}$ values of MG-X in this study are mainly determined by their $V_{\text {mes }} / V_{\mathrm{t}}$ or $V_{\text {mes }} / V_{\text {mic }}$ (see Table 1), and the higher the $V_{\text {mes }} / V_{\mathrm{t}}$ or $V_{\text {mes }} / V_{\text {mic }}$ is, the lower the $R_{\mathrm{ct}}$ value will be. At the low-frequency region, the curves are almost parallel to the ordinate, implying the ideal supercapacitor capacitive [39]. As shown in Figure 5(b), MC-1, MC-3, and MC-5 exhibit the Rs of about $0.38 \Omega, 0.25 \Omega$, and $0.23 \Omega$, which are in accordance with their higher oxygen content compared with MG-X, leading to an inferior specific capacitance correspondingly. Furthermore, for MC-X materials, the lower the oxygen content is, the lower the Rs will be, but the rate capability has an opposite trend. 


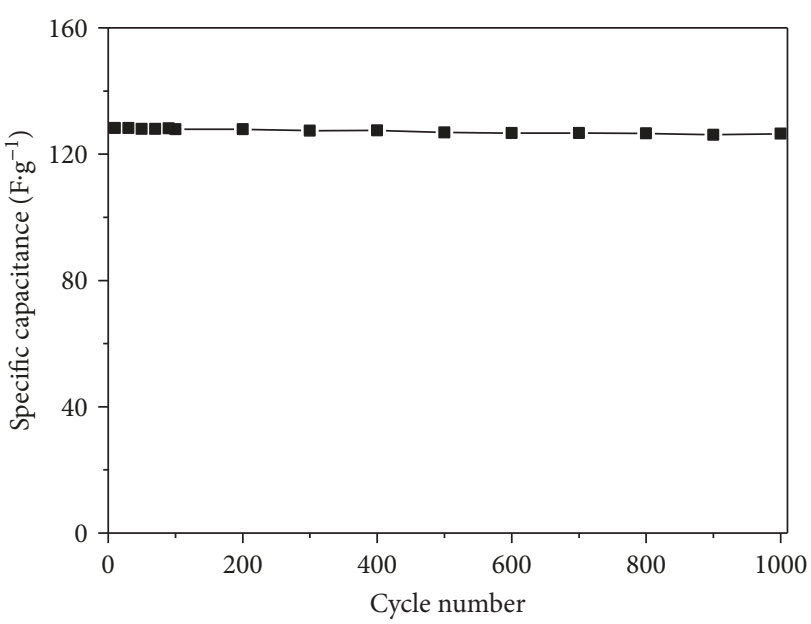

Figure 6: Cycling performance of MG-5 as electrode.

Figure 6 shows the relationship between specific capacitance and cycle number of MG-5 at $2.46 \mathrm{~A} \cdot \mathrm{g}^{-1}$. It can be found that MG-5 as electrode shows good cycling stability and high capacitance retention of $98.6 \%$ at 1000 th/1th discharge, which is comparable to those of porous graphene prepared by $\mathrm{KOH}$ activation (98.5\%) [23] and hierarchical porous carbon $/ \mathrm{MnO}_{2}$ composites (96\%) [40]. The good cycling stability is also based on its high mesoporosity and low electrochemical impedance.

\section{Conclusions}

In summary, a facile method was proposed to prepare mesoporous graphene materials from the potassium humate coupled with magnesium acetate. The MgO particles and freeze-drying pretreatment both have significant effects on the morphology and pore structure of MG-X. Moreover, MG-X exhibit a reduced oxygen content with enhanced $\mathrm{sp}^{2}$ carbon domain, large $S_{\mathrm{BET}}$, and high mesoporosity. As electrodes materials for supercapacitors, MG-X exhibit optimized electrochemical performance including high gravimetric capacitance $\left(150 \mathrm{~F} \cdot \mathrm{g}^{-1}\right.$ at $\left.3.3 \mathrm{~A} \cdot \mathrm{g}^{-1}\right)$, enhanced rate capability with the capacitance retention of $\sim 80 \%$ from 0.041 to $3.3 \mathrm{~A} \cdot \mathrm{g}^{-1}$, and good cycling performance. This work can pave a smart way to synthesize the mesoporous graphene materials for high-performance supercapacitors.

\section{Data Availability}

The data used to support the findings of this study are available from the corresponding author upon request.

\section{Conflicts of Interest}

The authors declare that there is no conflict of interest regarding the publication of this paper.

\section{Acknowledgments}

This work was financially supported by the National Natural Science Foundation of China (U1361119, 51404098), Key Scientific Research Project of Colleges and Universities in Henan Province (18A440002), and the Foundation of Henan Polytechnic University for Ph. D (B2014-008).

\section{Supplementary Materials}

Table S1: pore structure parameters of MC-X. Figure S1: N2 adsorption-desorption isotherms (a) and pore size distribution curves (b) of MC-X. Figure S2: Raman spectrum of MC. (Supplementary Materials)

\section{References}

[1] D. Zhu, Y. Wang, W. Lu et al., "A novel synthesis of hierarchical porous carbons from interpenetrating polymer networks for high performance supercapacitor electrodes," Carbon, vol. 111, pp. 667-674, 2017.

[2] H. Jeon, J. H. Han, D. M. Yu, J. Y. Lee, T. H. Kim, and Y. T. Hong, "Synthesis of mesoporous reduced graphene oxide by Zn particles for electrodes of supercapacitor in ionic liquid electrolyte," Journal of Industrial and Engineering Chemistry, vol. 45, pp. 105-110, 2017.

[3] H. Huang, Y. Tang, L. Xu, S. Tang, and Y. du, "Direct formation of reduced graphene oxide and 3D lightweight nickel network composite foam by hydrohalic acids and its application for high-performance supercapacitors," ACS Applied Materials \& Interfaces, vol. 6, no. 13, pp. 10248-10257, 2014.

[4] Z. Xu and C. Gao, "Graphene chiral liquid crystals and macroscopic assembled fibres," Nature Communications, vol. 2, no. 1, p. 571, 2011

[5] J. Zhu, D. Yang, Z. Yin, Q. Yan, and H. Zhang, "Graphene and graphene-based materials for energy storage applications," Small, vol. 10, no. 17, pp. 3480-3498, 2014.

[6] B. Wang, Y. Qin, W. Tan, Y. Tao, and Y. Kong, "Smartly designed 3D N-doped mesoporous graphene for highperformance supercapacitor electrodes," Electrochimica Acta, vol. 241, pp. 1-9, 2017.

[7] X. Yang, J. Zhu, L. Qiu, and D. Li, "Bioinspired effective prevention of restacking in multilayered graphene films: towards the next generation of high-performance supercapacitors," Advanced Materials, vol. 23, no. 25, pp. 2833-2838, 2011.

[8] K. Xia, Q. Li, L. Zheng et al., "Controllable fabrication of 2D and $3 \mathrm{D}$ porous graphene architectures using identical thermally exfoliated graphene oxides as precursors and their application as supercapacitor electrodes," Microporous and Mesoporous Materials, vol. 237, pp. 228-236, 2017.

[9] T. Kim, G. Jung, S. Yoo, K. S. Suh, and R. S. Ruoff, "Activated graphene-based carbons as supercapacitor electrodes with macro- and mesopores," ACS Nano, vol. 7, no. 8, pp. 68996905, 2013.

[10] L. L. Zhang, X. Zhao, M. D. Stoller et al., "Highly conductive and porous activated reduced graphene oxide films for highpower supercapacitors," Nano Letters, vol. 12, no. 4, pp. 1806-1812, 2012.

[11] Y. Zhu, S. Murali, M. D. Stoller et al., "Carbon-based supercapacitors produced by activation of graphene," Science, vol. 332, no. 6037 , pp. 1537-1541, 2011. 
[12] Z. Fan, Q. Zhao, T. Li et al., "Easy synthesis of porous graphene nanosheets and their use in supercapacitors," Carbon, vol. 50, no. 4, pp. 1699-1703, 2012.

[13] Y. Tian, Z. Wei, K. Zhang et al., "Three-dimensional phosphorus-doped graphene as an efficient metal-free electrocatalyst for electrochemical sensing," Sensors and Actuators B: Chemical, vol. 241, pp. 584-591, 2017.

[14] R. Zhang, H. An, Z. Li, M. Shao, J. Han, and M. Wei, "Mesoporous graphene-layered double hydroxides free-standing films for enhanced flexible supercapacitors," Chemical Engineering Journal, vol. 289, pp. 85-92, 2016.

[15] W. Yuan, L. Cheng, Y. Zhang et al., "Mesoporous nitrogendoped graphene aerogels with enhanced rate capability towards high performance supercapacitors," Ceramics International, vol. 43, no. 15, pp. 11563-11568, 2017.

[16] C. Powell and G. W. Beall, "Graphene oxide and graphene from low grade coal: synthesis, characterization and applications," Current Opinion in Colloid \& Interface Science, vol. 20, no. 5-6, pp. 362-366, 2015.

[17] E. M. Duraia and G. W. Beall, "Large temperature-induced red shift of G-band of functionalized graphene nanosheets synthesized from humic acid," Superlattices and Microstructures, vol. 98, pp. 379-384, 2016.

[18] E. M. Duraia, B. Henderson, and G. W. Beall, "Reduced humic acid nanosheets and its uses as nanofiller," Journal of Physics and Chemistry of Solids, vol. 85, pp. 86-90, 2015.

[19] G. Huang, Q. Liu, W. Kang, B. Xing, L. Chen, and C. Zhang, "Potassium humate based reduced graphite oxide materials for supercapacitor applications," Electrochimica Acta, vol. 196, pp. 450-456, 2016.

[20] J. Qin, S. Zhu, C. Feng et al., "In-situ space-confined catalysis for fabricating 3D mesoporous graphene and their capacitive properties," Applied Surface Science, vol. 433, pp. 568-574, 2018.

[21] X. Ma and D. Gao, "High capacitive storage performance of sulfur and nitrogen codoped mesoporous graphene," ChemSusChem, vol. 11, no. 6, pp. 1048-1055, 2018.

[22] L. Peng, Z. Xu, Z. Liu et al., "An iron-based green approach to 1-h production of single-layer graphene oxide," Nature Communications, vol. 6, no. 1, p. 5716, 2015.

[23] G. Wang, X. Shen, J. Yao, and J. Park, "Graphene nanosheets for enhanced lithium storage in lithium ion batteries," Carbon, vol. 47, no. 8, pp. 2049-2053, 2009.

[24] W. Shi, H. Fan, S. Ai, and L. Zhu, "Preparation of fluorescent graphene quantum dots from humic acid for bioimaging application," New Journal of Chemistry, vol. 39, no. 9, pp. 7054-7059, 2015.

[25] B. Xing, G. Huang, Z. Chen, L. Chen, G. Yi, and C. Zhang, "Facile preparation of hierarchical porous carbons for supercapacitors by direct carbonization of potassium humate," Journal of Solid State Electrochemistry, vol. 21, no. 1, pp. 263271, 2017.

[26] Y. Zhang, C. Zhang, G. Huang, B. Xing, and Y. Duan, "Tailoring the textural properties of hierarchical porous carbons for supercapacitors," Materials Letters, vol. 159, pp. 377-380, 2015.

[27] M. Dhelipan, A. Arunchander, A. K. Sahu, and D. Kalpana, "Activated carbon from orange peels as supercapacitor electrode and catalyst support for oxygen reduction reaction in proton exchange membrane fuel cell," Journal of Saudi Chemical Society, vol. 21, no. 4, pp. 487-494, 2017.
[28] J. Tian, C. Cui, C. Zheng, and W. Qian, "Mesoporous tubular graphene electrode for high performance supercapacitor," Chinese Chemical Letters, vol. 29, no. 4, pp. 599-602, 2018.

[29] M. Kota and H. S. Park, "Restacking-inhibited nitrogenincorporated mesoporous reduced graphene oxides for high energy supercapacitors," Ceramics International, vol. 44, no. 3, pp. 3195-3200, 2018.

[30] K. Gupta and O. P. Khatri, "Reduced graphene oxide as an effective adsorbent for removal of malachite green dye: plausible adsorption pathways," Journal of Colloid and Interface Science, vol. 501, pp. 11-21, 2017.

[31] Y. C. G. Kwan, G. M. Ng, and C. H. A. Huan, "Identification of functional groups and determination of carboxyl formation temperature in graphene oxide using the XPS O 1s spectrum," Thin Solid Films, vol. 590, pp. 40-48, 2015.

[32] P. S. Das, S. Bakuli, I. Biswas et al., "RGO/MgO hybrid nanocomposites with high specific capacitance," Ceramics International, vol. 44, no. 1, pp. 424-432, 2018.

[33] X. Wang, Y. Zhang, C. Zhi et al., “Three-dimensional strutted graphene grown by substrate-free sugar blowing for highpower-density supercapacitors," Nature Communications, vol. 4, no. 1, pp. 2905-2908, 2013.

[34] C. Long, X. Chen, L. Jiang, L. Zhi, and Z. Fan, "Porous layerstacking carbon derived from in-built template in biomass for high volumetric performance supercapacitors," Nano Energy, vol. 12, pp. 141-151, 2015.

[35] S. Ye and J. Feng, "Self-assembled three-dimensional hierarchical graphene/polypyrrole nanotube hybrid aerogel and its application for supercapacitors," ACS Applied Materials \& Interfaces, vol. 6, no. 12, pp. 9671-9679, 2014.

[36] C. Zheng, X. F. Zhou, H. L. Cao, G. H. Wang, and Z. P. Liu, "Controllable synthesis of activated graphene and its application in supercapacitors," Journal of Materials Chemistry A, vol. 3, no. 18, pp. 9543-9549, 2015.

[37] G. Zhao, C. Chen, D. Yu et al., "One-step production of O-N-S co-doped three-dimensional hierarchical porous carbons for high-performance supercapacitors," Nano Energy, vol. 47, pp. 547-555, 2018.

[38] B. Chang, H. Yin, X. Zhang, S. Zhang, and B. Yang, "Chemical blowing strategy synthesis of nitrogen-rich porous graphitized carbon nanosheets: morphology, pore structure and supercapacitor application," Chemical Engineering Journal, vol. 312, pp. 191-203, 2017.

[39] Y. Gao, W. Zhang, Q. Yue et al., "Simple synthesis of hierarchical porous carbon from Enteromorpha prolifera by a selftemplate method for supercapacitor electrodes," Journal of Power Sources, vol. 270, pp. 403-410, 2014.

[40] Y. Zhang, C. Zhang, G. Huang, B. Xing, and Y. Duan, "Synthesis and capacitive properties of manganese oxide nanoparticles dispersed on hierarchical porous carbons," Electrochimica Acta, vol. 166, pp. 107-116, 2015. 


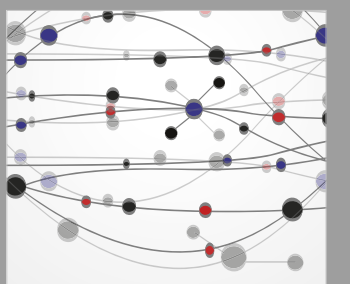

The Scientific World Journal
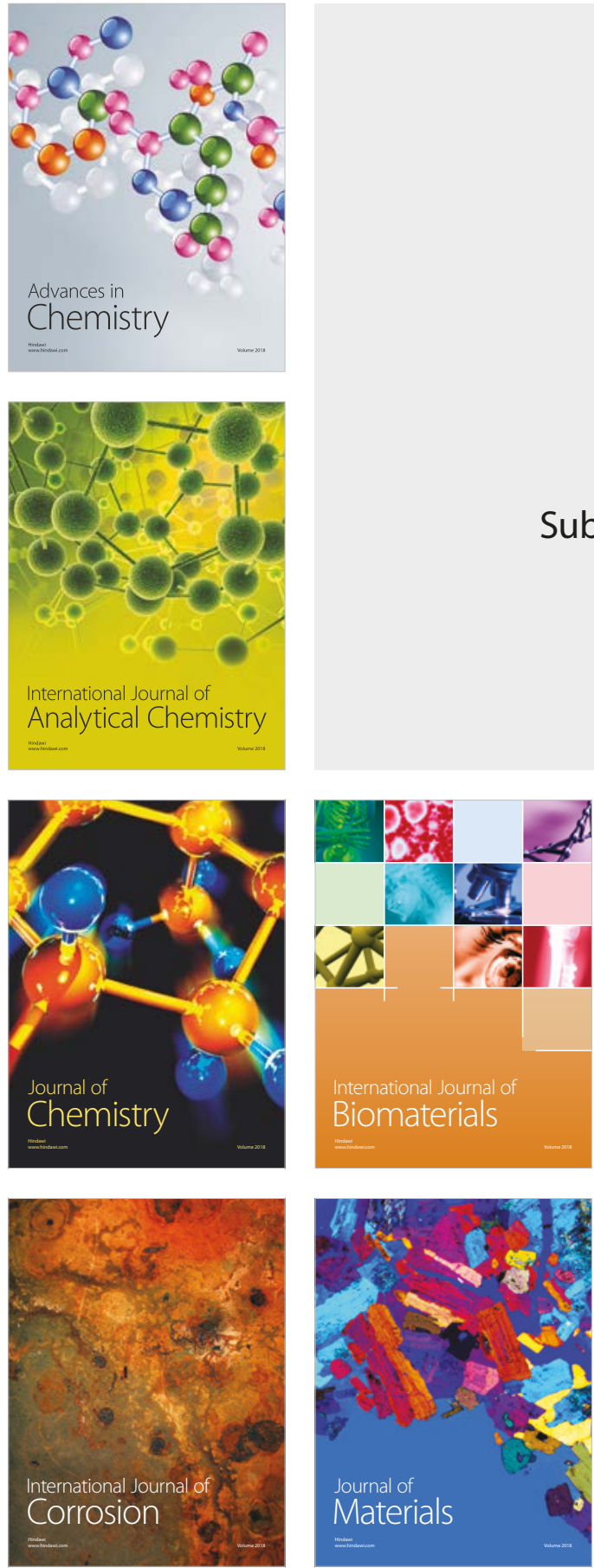

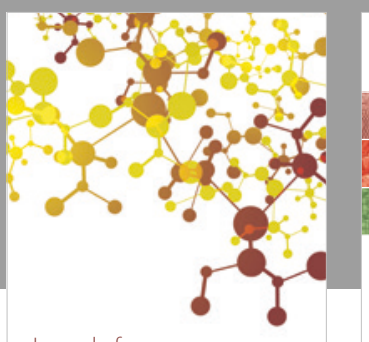

Journal of

Applied Chemistry
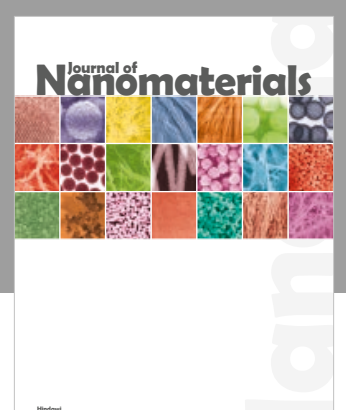

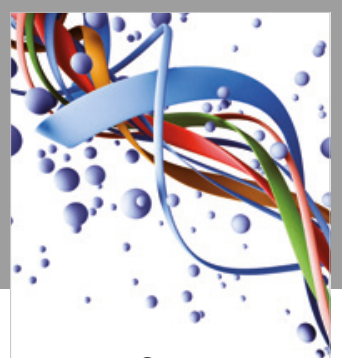

Scientifica

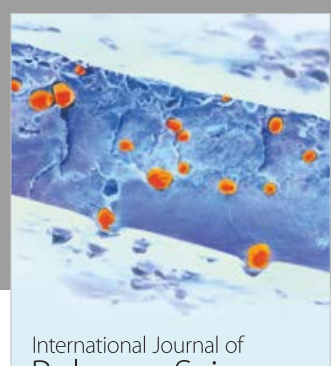

Polymer Science

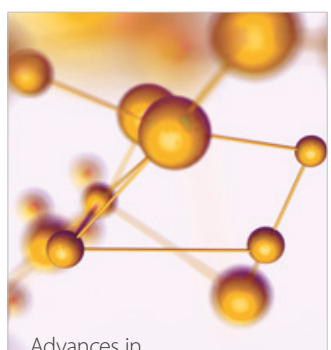

Physical Chemistry
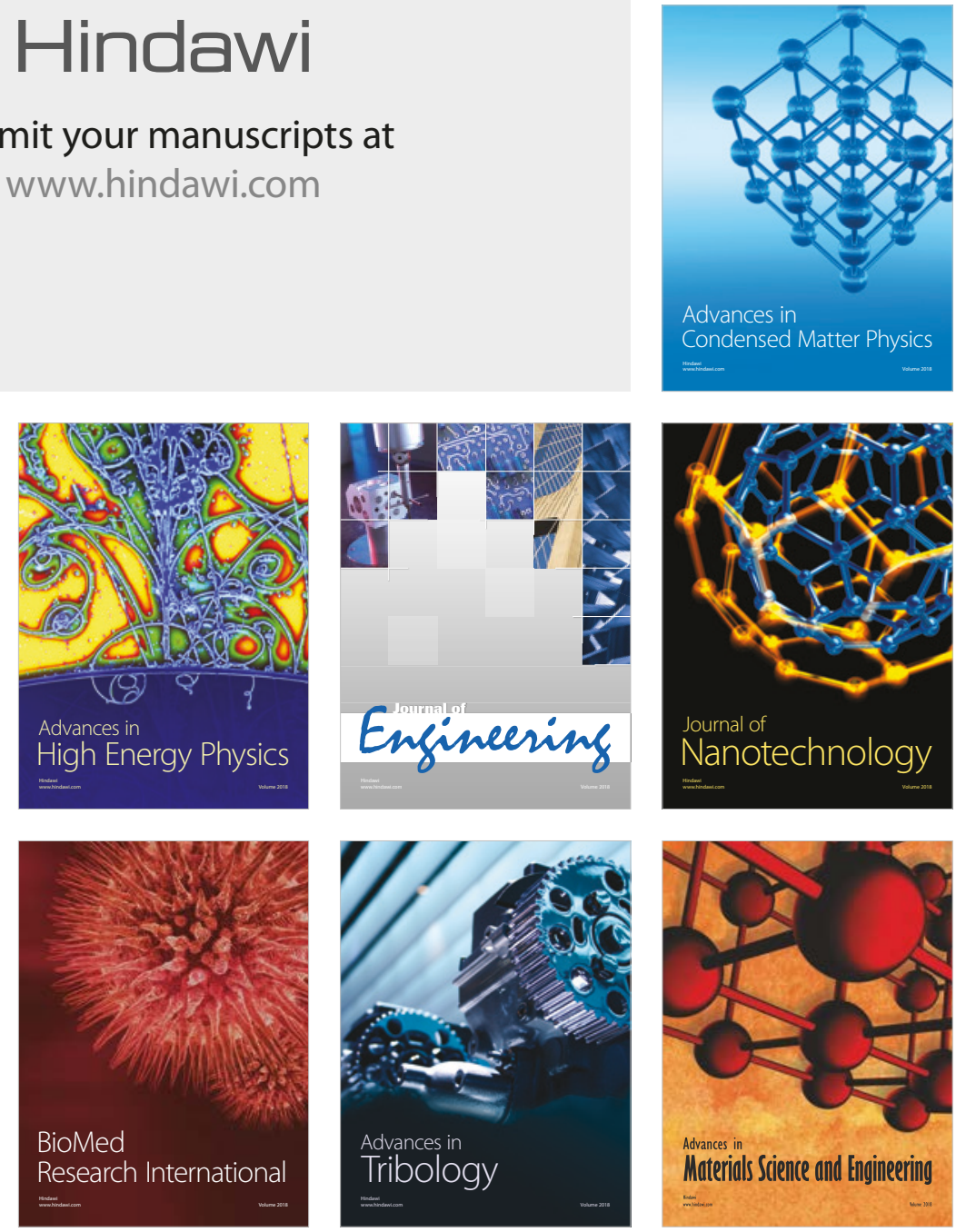